University of Nebraska - Lincoln

DigitalCommons@University of Nebraska - Lincoln

Biological Systems Engineering: Papers and

Publications

Biological Systems Engineering

2006

Circular Ultrasound Compounding by Designed Matrix Weighting

Gregory Bashford

University of Nebraska-Lincoln, gbashford2@unl.edu

Jonathan Morse

University of Nebraska-Lincoln

Follow this and additional works at: https://digitalcommons.unl.edu/biosysengfacpub

Part of the Biological Engineering Commons

Bashford, Gregory and Morse, Jonathan, "Circular Ultrasound Compounding by Designed Matrix Weighting" (2006). Biological Systems Engineering: Papers and Publications. 39.

https://digitalcommons.unl.edu/biosysengfacpub/39

This Article is brought to you for free and open access by the Biological Systems Engineering at DigitalCommons@University of Nebraska - Lincoln. It has been accepted for inclusion in Biological Systems Engineering: Papers and Publications by an authorized administrator of DigitalCommons@University of Nebraska Lincoln. 


\title{
Circular Ultrasound Compounding by Designed Matrix Weighting
}

\author{
Gregory R. Bashford*, Senior Member, IEEE, and Jonathan L. Morse
}

\begin{abstract}
Spatial compounding is an imaging technique that aims to improve image contrast by combining partially decorrelated images acquired at different angles or positions. In conventional spatial compounding, data sets are combined with equal weighting. Here, we describe an alternative method of reconstruction using algorithms which weight the data based on a "quality" matrix. The quality matrix is derived from beamforming characteristics. For each data set, the reliability of the data is assumed to vary spatially. By compounding the data based on the quality matrix, a complete image is formed. Here, we describe the construction of a rotational translation stage and tissue-mimicking phantoms that are used in conjunction with a commercial medical ultrasound machine to test our reconstruction algorithms. The new algorithms were found to increase the contrast-to-speckle ratio of simulated cysts and tumors by $61 \%$ from raw data, and to significantly increase edge definition of small embedded targets. The new method shows promise as a computationally efficient method of improving contrast and resolution in ultrasound images. The method should be particularly useful in breast imaging, where images from multiple angles can be acquired without interference from bone or air.
\end{abstract}

Index Terms-Breast imaging, cancer screening, medical imaging, spatial compounding, ultrasound.

\section{INTRODUCTION}

\section{A. Clinical Relevance}

Other than skin cancer, breast cancer is the most common cancer among women in the United States. Early detection of breast cancer through screening has been shown to reduce the risk of dying from breast cancer [1]. Currently, the best tool available for screening is the X-ray mammogram, which can detect some tumors before they are palpable. The National Cancer Institute and most doctors recommend that women in their forties and older be screened by X-ray mammogram every 1-2 years [2]. Screening may be defined as detection of disease in a population at risk but in which disease is not suspected. Criteria for effective screening include: a simple reproducible test, few complications, inexpensive, noninvasive, high sensitivity for detection, detected disease is effectively treatable, and by which early detection saves lives. Only X-ray mammography has been

Manuscript received December 15, 2005; revised January 24, 2006. This work was supported in part by the University of Nebraska Agricultural Research Division Journal Series 14821. Asterisk indicates corresponding author.

*G. R. Bashford is with the Department of Biological Systems Engineering, University of Nebraska, Lincoln, NE 68583 USA (e-mail: gbashford2@unl. edu).

J. L. Morse was with the University of Nebraska, Lincoln, NE 68583 USA. He is now with the Department of Electrical Engineering, Massachusetts Institute of Technology, Cambridge, MA 02139 USA (e-mail: jlmorse@mit.edu).

Digital Object Identifier 10.1109/TMI.2006.873610 shown over many years and population studies to be an effective screening imaging modality for breast cancer detection, fulfilling the criteria above.

In breast imaging, a diagnostic is an in-depth imaging study of a previously found or highly suspected abnormality. Current accepted techniques utilize special X-ray mammographic techniques (e.g., magnification radiography), conventional ultrasound imaging, or magnetic resonance imaging (MRI). Ultrasound imaging is the only modality to use mechanical (pressure) waves as the energy source (in contrast to X-rays, which are a form of electromagnetic energy). Ultrasound is a reflection imaging modality, meaning the reflections of sound sent into the body are measured and displayed as an image. In conventional ultrasound, the energy is beamformed (steered and focused) into the body through electronic phasing of individual transducer array elements. There are two major advantages of ultrasound. The first is that real-time data (15-30 frames/s) may be viewed. This is due to the relative ease (electronically) in acquiring and displaying ultrasonic signals. For example, heart valve dynamics and fetal movements may be visualized "live." The second is that nonionizing energy (incapable of removing bound electrons from atomic shells) is used, thereby substantially reducing the risk to the patient. Ultrasound is also painless, relatively inexpensive, and can detect masses even in the radio-dense breast.

Currently, conventional ultrasound meets the standards of an effective diagnostic technique, but it has not been shown to meet the criteria for an effective screening technique. Usually, ultrasound cannot conclusively distinguish solid malignant from solid benign tissue. Confidence in complete inclusion of all breast tissue is more difficult than in X-ray mammography. In addition, ultrasound has not been shown to reliably detect breast microcalcifications. For these reasons, conventional ultrasound has been limited to determining whether detectable cysts are solid or fluid [3]. Researchers have attempted to define the engineering specifications for ultrasound to have the potential for effective breast cancer screening. To be clinically useful, the images should have a resolution approaching $1 \mathrm{~mm}$ for precancerous lesion detection, be able to discriminate tumors from surrounding soft tissue, and have sensitivity sufficient to detect microcalcifications [4].

\section{B. Spatial Compounding}

One reason ultrasound not often meets these requirements is the presence of coherent wave interference, or speckle, which produces a "grainy" appearance to the image and artificially reduces the perceived resolution [5]. Another reason is the presence of clutter, which is comprised of echoes from side lobes, grating lobes, and multipath reverberations. A third reason is 

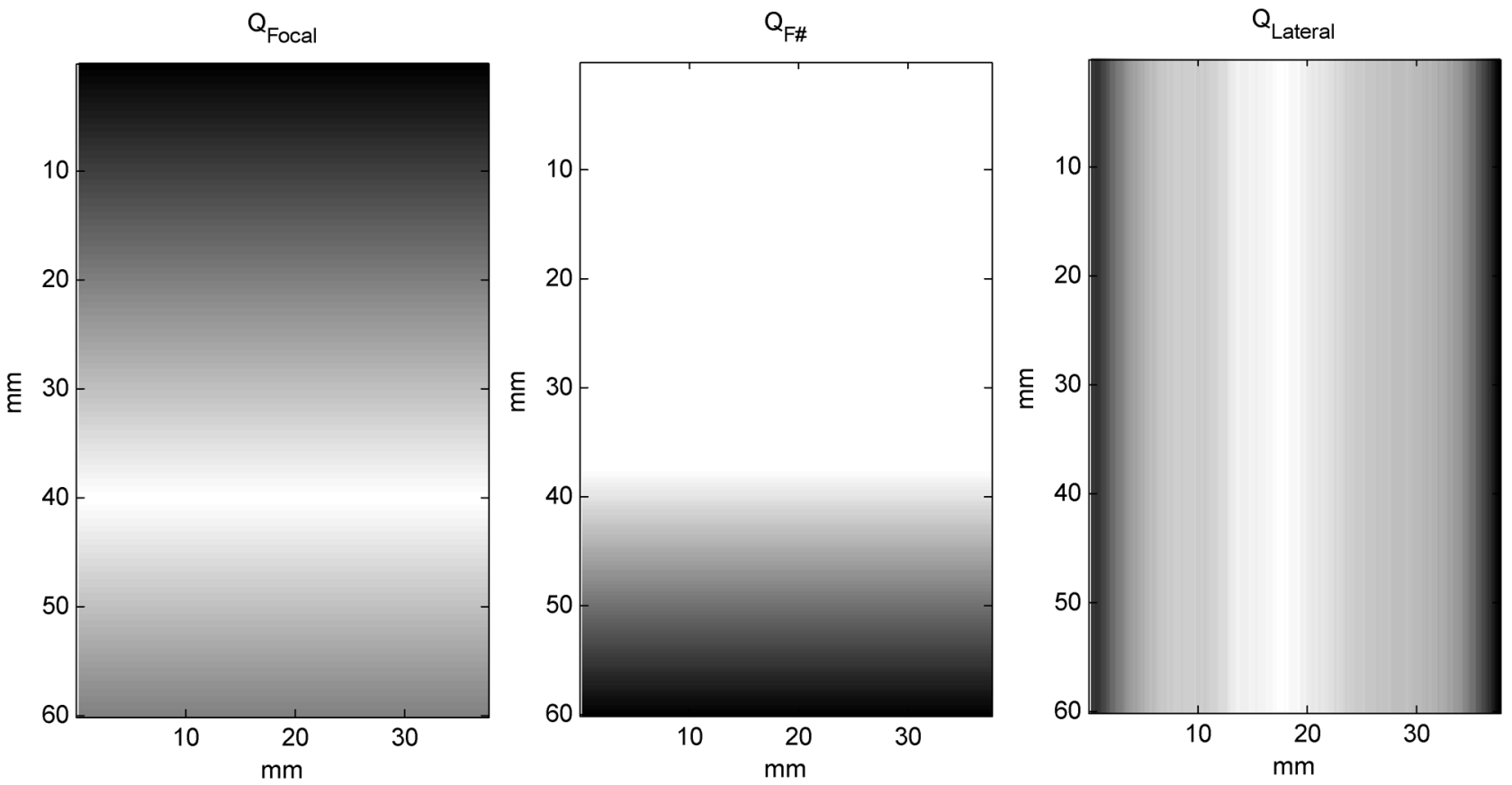

Fig. 1. Quality matrices. The figures depict the relative value of data contained within a $60 \mathrm{~mm} \times 37 \mathrm{~mm}$ image. (left) (Q $\mathrm{Q}_{\text {Focal }}$ ), the data corresponding to pixels near the focal zone (at $4 \mathrm{~cm}$ ) are given a higher relative weight (indicated by brighter intensity). (center) $\left(\mathrm{Q}_{\mathrm{F} \#}\right)$, the data corresponding to pixels within an $\mathrm{F} / 1$ region are given equal weight, with decreasing weight given to pixels at further ranges. (right) ( $\left.\mathrm{Q}_{\text {Lateral }}\right)$, the data nearer the middle of the image is weighted more strongly than data at the sides.

due to anisotropic reflections, including specular (mirror-like) reflectors, which produce stronger echoes at angles nearly perpendicular to the acoustic beam axis than angles tilted off-axis. Collectively, speckle, clutter, and specular reflectors obstruct visualization of cysts, solid lesions, and microcalcifications. Spatial compounding is a technique that aims to reduce this obstruction by averaging images taken at different (but overlapping) spatial positions. Normally, the spatial positions differ by angle of insonation. Spatial compounding is of particular interest in breast imaging, where images from a broad range of angles can be obtained. The absence of bone or air in the breast increases the area of overlap that can be achieved.

Spatial compounding relies on consistency of desired reflectivity patterns and uncorrelated image artifacts across view angles. Under these conditions, image artifacts are suppressed, while desired patterns preserved during averaging. Spatial compounding has consistently shown marked improvement in the contrast and perceived resolution of ultrasound images [3], [6]-[8]. However, standard spatial compounding does not distinguish between individual raw data sets that are averaged. Instead, the raw data sets are equally weighted when averaging. It is hypothesized that since the local resolution and contrast vary in an ultrasound image due to beamforming effects, consideration of these parameters will increase the compounded resolution and contrast.

\section{Objectives}

In this paper, we propose an alternative strategy for the reconstruction of a composite image from several data sets differing by acquisition angle. The new strategy is similar to spatial compounding; however, the individual data sets are weighted based on a "quality matrix" before averaging. In simple averaging, each data set is implicitly considered to be of equal value. In the new method, specific areas of the data set (based on beamforming theory) are explicitly assigned a greater relative value. The objectives of this paper are to introduce the method, and compare its performance to standard spatial compounding and conventional image formation without compounding on threedimensional tissue phantoms containing simulated cysts and tumors, and embedded small targets. The image parameters to be compared are contrast (of simulated cysts and tumors) and target size (of small embedded glass beads).

\section{THEORY}

\section{A. New Compounding Method}

The quality matrix is a numerical array of equal size to the raw data set (individual B-mode images). The elements of this matrix represent the relative quality of the corresponding pixels in the raw data set. Here, "quality" refers to an image quality parameter such as resolution or contrast. For example, the area of the raw image near the known transmit focus possesses better spatial resolution than areas further away from the transmit focus. Therefore, in each raw data set, pixels corresponding to the area near the transmit focus are assigned a larger relative quality value than pixels corresponding to areas away from the transmit focus. The quality matrix can be visualized as an image, with increasing brightness corresponding to higher quality assignments. In general, the creation of a quality matrix consists of identifying a beamforming parameter to weight and assign values. The amplitude of the quality values is rescaled as a last step to achieve weights from 1 to 10 . In the work described here, four quality matrices, and combinations thereof, were used in reconstruction. Three of the quality matrices are shown in Fig. 1. 


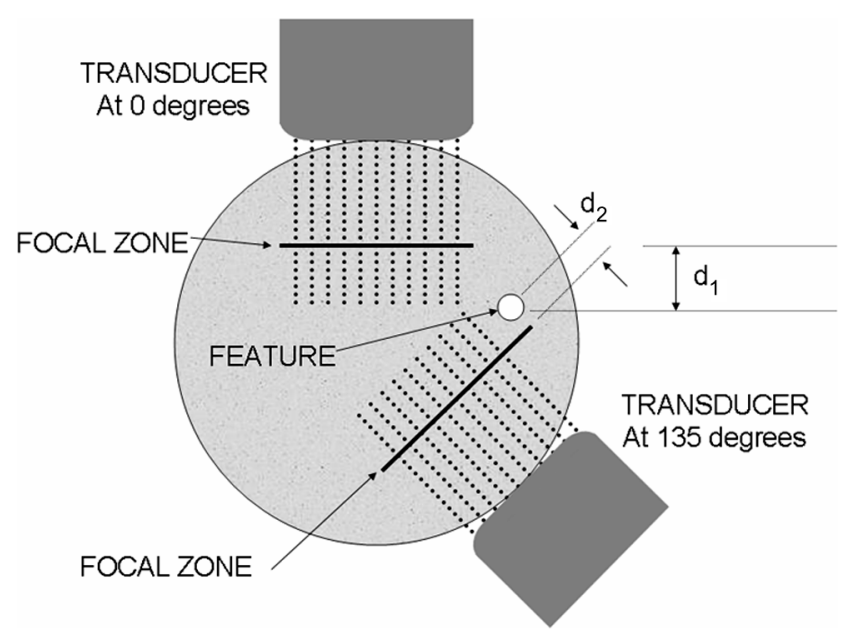

Fig. 2. Example quality derivation. The angular reflection data is weighted according to the distance from the focal zone. In the figure, $d_{2}<d_{1}$, therefore data taken at $135^{\circ}$ is weighted more for pixels corresponding to the feature.

The first matrix $\left(\mathrm{Q}_{1}\right.$, not shown) is a matrix of ones, essentially creating an unweighted average. This is equivalent to standard spatial compounding, with the assumption that nonoverlapping regions are not averaged. The second matrix (QFocal) weights data linearly by its distance from the focal zone. The third matrix $\left(\mathrm{Q}_{\mathrm{F} \#}\right)$ is formed by assigning pixels up to a depth equal to the width of the transducer $(\mathrm{F} / 1)$ the same value, with a linear decrease from that point on. The rationale is that the commercial machine is able to achieve a constant $\mathrm{F} \#$ (focal distance divided by the width of element group used to form the image) up to that depth, and decreasing thereafter. Here, pixels at the bottom of the image, or the farthest from the transducer, were given low priority. The fourth matrix $\left(\mathrm{Q}_{\text {Lateral }}\right)$ takes into consideration pixels near the sides of the image versus those near the center. Pixels near the edge are given a lower quality weight due to the natural decrease in $\mathrm{F} \#$ from fewer transducer elements being used near the edge of the image. This matrix is formed by averaging together all 90 raw data sets, then taking the mean value down the columns. The resulting signal is used to weight the fourth quality matrix laterally. Here, it is assumed that in general a brighter signal corresponds to better resolution [9]. Individual quality matrices or combinations of quality matrices (formed by multiplication) were used in reconstruction.

The final image is reconstructed on a pixel-by-pixel basis. For each pixel in the final image, several data sets may be used to derive the final intensity value. Each data set used for the given pixel will contribute differently depending on the quality of the data contained in the data set. This method is designed to be simple and computationally efficient. An example is the best way to clarify this process. Consider the scanning configuration in Fig. 2.

A circular target is scanned by a transducer, which possesses a certain transmit focus. Each data set is obtained by conventional beamforming, producing an envelope-detected B-mode image. After each image acquisition, the transducer assembly is rotated to a new angle where a new data set is obtained. When all data sets have been acquired, reconstruction takes place on a pixel-by-pixel basis. The particular feature indicated in the target is closer to the focal zone when the transducer is in the $135^{\circ}$ position than it is in the $0^{\circ}$ position. When reconstructing the pixels corresponding to the feature location, both data sets will be used, but the data taken at the $135^{\circ}$ location will be weighted more heavily than the data taken at the $0^{\circ}$ location. The rationale is that data taken closer to the focal zone has better lateral resolution than data taken further away from the transducer. (Of course, all data sets, not just two, will be used). The relative weighting amount will depend on the particular quality matrix assigned to the data sets.

It is important to note that the process of compounding weighted data sets is different than image gain compensation. In gain compensation, systems engineers attempt to create an overall uniform brightness across the image by adjusting the gain as a function of both distance (correcting for tissue attenuation, beam shape, expected anatomical features, etc.) and space (increased gain at the edges of the image due to less elements being used). In the new method, the raw data sets have already been through gain compensation since they are extracted from image files (see Methods below). The shape of the quality matrices (e.g., linear) in this paper is not intended to correct for the nonlinear signal amplitudes seen by the beamformer. Rather, the compounding method described here assumes that gain compensation has been previously completed.

\section{B. Quality Derivation}

The weighting of individual data sets proposed here is based on a simple quality metric. Specifically, $N$ values $\left[x_{1} x_{2} \ldots x_{N}\right]$, one from each of the raw data sets are used to reconstruct a single intensity value in the final image. Each value has a corresponding quality value $q_{i}$, and the final intensity value $x_{\text {final }}$ is calculated as

$$
x_{\text {final }}=\frac{\sum_{i=1}^{N} x_{i} q_{i}}{\sum_{i=1}^{N} q_{i}} .
$$

This centroid-type calculation weights individual values according to a predefined quality value. The quality values are unitless, being a relative measure of priority with other pixel locations in the data set. When assigning quality values, prior knowledge about the scanning configuration is needed in order to weight different areas of the raw data accordingly. In the example given above, areas near the focal zone are given higher weight. Other examples may be a distance from the transducer, such as depth-dependent F\#, or poor contact with the target (see below). The computation is self-normalizing; therefore, the only condition on the quality matrix is that it be self-consistent.

\section{Reconstruction Algorithm}

In the reconstruction algorithm developed here, it is assumed that each data set consists of a rectangular region (as would be acquired from a B-mode imaging device with a linear array), as shown in Fig. 3.

A particular data set is shown at scan angle $\theta$. The data set is orthogonal to a rotated axes set $x^{\prime}$ and $y^{\prime}$. Within this data set, a particular B-mode intensity value lies at point $(r, c)$ in matrix 


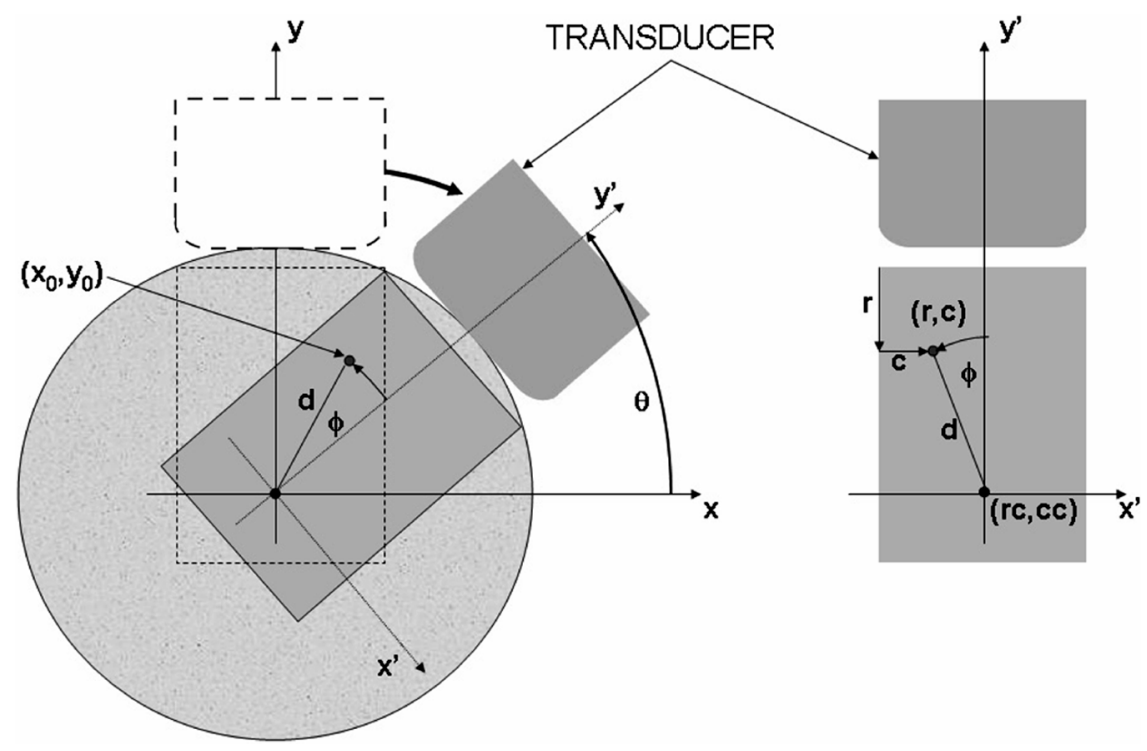

Fig. 3. Reconstruction geometry. (left) Diagram showing scan coordinates within the circular target. (right) Scan coordinates on rotated axes.

coordinates. This intensity value will be used for the reconstruction value at point $\left(x_{0}, y_{0}\right)$ in the final image. This same point lies at a distance $d$ from the rotation center at an angle $\phi$ with the rotated axes $x^{\prime}$ and $y^{\prime}$. Since MATLAB is used to reconstruct the image, a simple conversion may be derived for matrix coordinates and the rotated axes. First, the coordinate $(d, \phi)$ in terms of the raw data matrix coordinates $(r, c)$ is

$$
\begin{aligned}
& d=\sqrt{(r c-r)^{2}+(c c-c)^{2}} \\
& \phi=\tan ^{-1}\left(\frac{c c-c}{r c-r}\right)
\end{aligned}
$$

where $r c$ is the center row, $c c$ is the center column, and a fourquadrant arctangent is used. The center of rotation can be within the data sets or outside of them. Then, any point in the final image $\left(x_{0}, y_{0}\right)$ can be expressed as

$$
\begin{aligned}
& x_{0}=d \cos (\theta+\phi) \\
& y_{0}=d \sin (\theta+\phi) .
\end{aligned}
$$

The size of the matrix needed to encompass all of the rotated data sets can be found by first considering the maximum distance from the rotation point a data set point may be

$$
d_{\max }=\operatorname{ceil}\left(\sqrt{(r c-1)^{2}+(c c-1)^{2}}\right)
$$

where "ceil" is the integer ceiling function. Accounting for the origin, the final image will fill a square matrix with $\left(2^{*} d_{\max }+1\right)$ elements on a side. Here, it is also assumed that matrix coordinates are numbered $1: N$ rather than $0:(N-1)$. Finally, in matrix coordinates, the position corresponding to $(r, c)$ in the final image $(n r, n c)$ will be

$$
\begin{aligned}
& n r=d_{\max }+1-y_{0} \\
& n c=d_{\max }+1-x_{0} .
\end{aligned}
$$

Reconstruction proceeds as follows: for each element in the raw data set for a particular scan angle, the position in the final image that the element corresponds to is calculated. The quality value corresponding to the element position is multiplied by the element intensity and added to the current contents. In a separate matrix of identical size to the final image, the quality value itself is added to the element at the same location. When all scan angles and all data points have been processed, the final image matrix is divided point-by-point by the quality sums.

\section{MATERIALS AND METHODS}

\section{A. Phantoms}

Ideal phantoms will accurately mimic the tissue properties of speed of sound and frequency-dependent attenuation. Two types of phantoms were created for this study. The first type was based on previously published recipes from Madsen's group [10]-[12]. These phantoms include ingredients which are controllable to mimic the speed of sound, attenuation, and backscatter in human tissue (generally to within $1 \%$ of desired values). The ingredients were selected to produce a phantom with speed of sound $=1520 \mathrm{~m} / \mathrm{s}$ (similar to breast tissue), and frequency-dependent attenuation $=0.5 \mathrm{~dB} / \mathrm{cm} / \mathrm{MHz}$. Table I shows a list of ingredients used for the first type of phantom.

The details of phantom construction are briefly given here. To make the phantom mold, the water and oil were brought to $\sim 70^{\circ} \mathrm{C}$ on separate hot plates. Then, the gelatin powder, $p$-methylbenzic acid powder, and $n$-propanol were added to the water and stirred in. The warm oil was then added to the gelatin beaker followed by the Ivory detergent. After the appropriate amount of stirring (approximately $2 \mathrm{~min}$ ) the scattering beads and formaldehyde were mixed in and the hot mixture was poured into the appropriate mold for congealing. Before the gelatin set, simulated cysts and tumors were added. The simulated cysts were a mixture of only water, gelatin, acid, and alcohol set inside a small water balloon, approximately 
TABLE I

IngREDIENTS IN TYPE 1 TISSUE PHANTOM

\begin{tabular}{llll}
\hline \hline \multicolumn{1}{c}{ Ingredient } & \multicolumn{1}{c}{ Concentration } & Amount Used & \multicolumn{1}{c}{ Reason for Use } \\
\hline reverse osmosis water & - & $225 \mathrm{~mL}$ & liquefy gelatin \\
n-propanol & $0.088(\mathrm{~mL} / \mathrm{mL})$ & $35.2 \mathrm{~mL}$ & control speed of sound \\
p-methylbenzoic acid & $0.002(\mathrm{~g} / \mathrm{mL})$ & $0.8 \mathrm{~g}$ & preservative \\
gelatin powder & $1 / 5 \mathrm{Gelatin} / \mathrm{Water}$ & $52.2 \mathrm{~g}$ & structure \\
olive oil & 0.204 & $81.6 \mathrm{~mL}$ & attenuation coefficient \\
castor oil & 0.136 & $54.4 \mathrm{~mL}$ & attenuation coefficient \\
ivory detergent & $33: 1 \mathrm{Oil} / \mathrm{Detergent}$ & $4.12 \mathrm{~mL}$ & emulsifier \\
formaldehyde & $13.4 \mathrm{~mL} / \mathrm{L}$ & $3 \mathrm{~mL}$ & gelatin crosslinker \\
40 micron glass beads & $0.11 \mathrm{mg} / \mathrm{cm}^{3}$ & $44 \mathrm{~g}$ & scatterers \\
\hline \hline
\end{tabular}

TABLE II

IngREDIENTS IN TYPE 2 TISSUE PHANTOM

\begin{tabular}{llll}
\hline \hline Ingredient & \multicolumn{1}{c}{ Concentration } & Amount Used & Reason for Use \\
\hline reverse osmosis water & - & $200 \mathrm{~mL}$ & liquefy gelatin \\
gelatin powder & $1 / 5 \mathrm{Gelatin} /$ Water & $40 \mathrm{~g}$ & structure \\
formaldehyde & $13.4 \mathrm{~mL} / \mathrm{L}$ & $2.6 \mathrm{~mL}$ & gelatin crosslinker \\
graphite Powder & $0.073 \mathrm{~g} / \mathrm{cm}^{3}$ & $14.6 \mathrm{~g}$ & scatterers \\
\hline
\end{tabular}

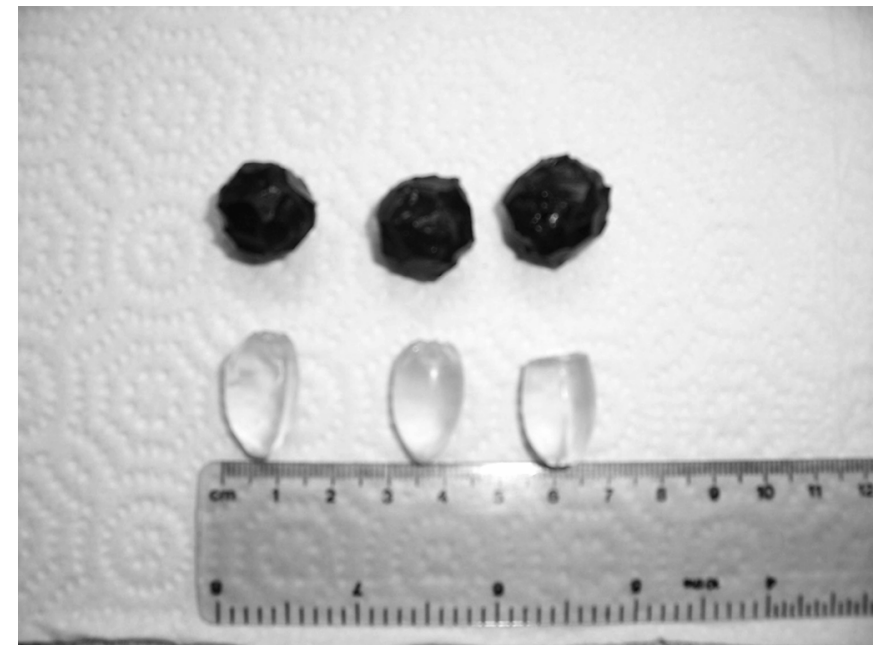

Fig. 4. Examples of simulated cysts (bottom) and tumors (top).

$10-15 \mathrm{~mm}$ in diameter when set. This gives a roughly spherical shape to the cyst (Fig. 4). The simulated tumors were made in a water balloon with a large amount of graphite powder $\left(0.11 \mathrm{~g} / \mathrm{cm}^{3}\right)$ added to the cyst substrate to make a spherical region of high scatterer concentration. The simulated cysts and tumors were added to the phantom, while the phantom material is still plastic enough to flow around the spheres, but solid enough so they do not sink following insertion. After noting the relative locations of the simulated cysts and tumors, a metal bracket attached to wires was pushed $1 \mathrm{~cm}$ into the top of the phantom. This was to suspend the phantom in the water tank from above. Finally, the phantom was allowed to fully set up in the mold overnight in the refrigerator.

The second type of phantom was a simpler mixture of gelatin, graphite (for scattering), and formaldehyde. These ingredients were based on a phantom also published previously [13]. The ingredients for this phantom are listed in Table II. The ingredients were selected to produce a phantom with speed of sound $=1550 \mathrm{~m} / \mathrm{s}$ and frequency-dependent attenuation $=0.5 \mathrm{~dB} / \mathrm{cm} / \mathrm{MHz}$. The method of production was similar to that of the first phantom. To the second phantom type were added 1.0 and $2.5-\mathrm{mm}$ diameter glass beads, to simulate small targets. The beads were added just before the phantom started to congeal, which allowed them to sink a few millimeters below the surface of the phantom.

\section{B. Rotating Stage}

A radial stage was designed (Fig. 5) to hold a commercial ultrasound transducer in one horizontal plane and rotate the transducer around a circular tissue-mimicking phantom. A Phase II rotary machining table forms the base of the unit. The table is $15 \mathrm{~cm}$ in diameter. The scan table rotates in conjunction with the transducer holding arm. This allows a water tank with an acoustic window to be placed upon the table and rotate with the transducer. The phantom is not placed upon the table but suspended into the water-filled tank from an overhead harness. In this manner, the transducer does not physically touch the target. Instead, it contacts an acoustically appropriate window in the water-filled tank wall. A circular standoff pad (Ultraphonic Focus, Pharmaceutical Innovations, Newark, NJ) was used as the window between the transducer and the water in the tank. The pad has a diameter of $9 \mathrm{~cm}$ and a thickness of $1.5 \mathrm{~cm}$. A custom built clamp sealed the pad to the tank wall to keep the water inside.

Rotary and vertical translation of the transducer arm are computer controlled through DC stepper motors (4034-329, Applied Motion Products, Watsonville, CA). The motors are 3 volt, $4 \mathrm{amp}$, microstepping, unipolar DC motors that require a control board (UCC3030, Peter Nordberg Consulting, Inc., Ferguson, MO) to operate with and interface to the computer. The vertical-translation motor is set at the bottom of a ball-screw shaft for precise vertical control. The benefit of the ball-screw over a traditional acme screw is the elimination of the "backlash" inherent with an acme screw. A LabVIEW (National Instruments, Austin, TX) program was written to interface with the motors. 


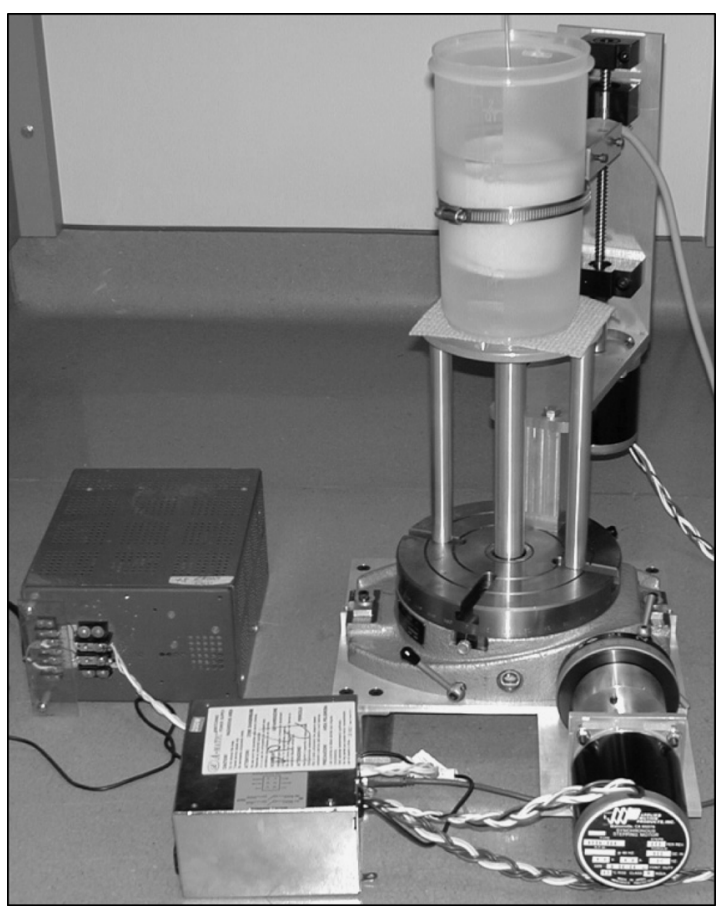

Fig. 5. Scanning tank sitting atop rotary table and scan assembly. The phantom is suspended in the tank from above.

\section{Scan Procedure}

The phantom was placed on the rotary table and centered on the holding platform. A commercial ultrasound machine (Siemens Antares) was used with the "V13-5" transducer set to $10.0 \mathrm{MHz}$ for imaging the phantom. The transducer was attached to the holder on the table and carefully set so that it contacted the standoff pad. Ultrasound coupling gel was applied to the transducer/pad interface to aid in contact throughout vertical translation. A $6 \mathrm{~cm}$ scan depth was used with one focal zone set at $4 \mathrm{~cm}$.

Images were acquired by the following process: The rotary table was set at $0^{\circ}$ and an image was saved on the ultrasound machine. The transducer was rotated $4^{\circ}$ clockwise around the phantom and another image was saved. This process was repeated for a total of 90 images around $360^{\circ}$. After one complete revolution, the transducer was moved vertically by $1 \mathrm{~mm}$ to acquire a new plane in the phantom. This process was repeated until the desired number of planes was acquired. The raw data were saved in DICOM format on the ultrasound machine's hard drive. The images were transferred to a PC, where software (DicomWorks, version 1.3.5) was used to convert the DICOM files into JPEG images. MATLAB was used for all image processing and reconstruction tasks.

\section{Image Quality Parameters}

Two image quality metrics, a contrast measure and a target diameter measure, were used to evaluate the effects of the quality matrices. First, cyst and tumor detectability was calculated by the contrast-to-speckle (CSR) ratio previously proposed and used by other investigators [14], [15]. This parameter is defined as

$$
\mathrm{CSR}=\frac{S_{o}-S_{i}}{\sqrt{\sigma_{o}^{2}+\sigma_{i}^{2}}}
$$

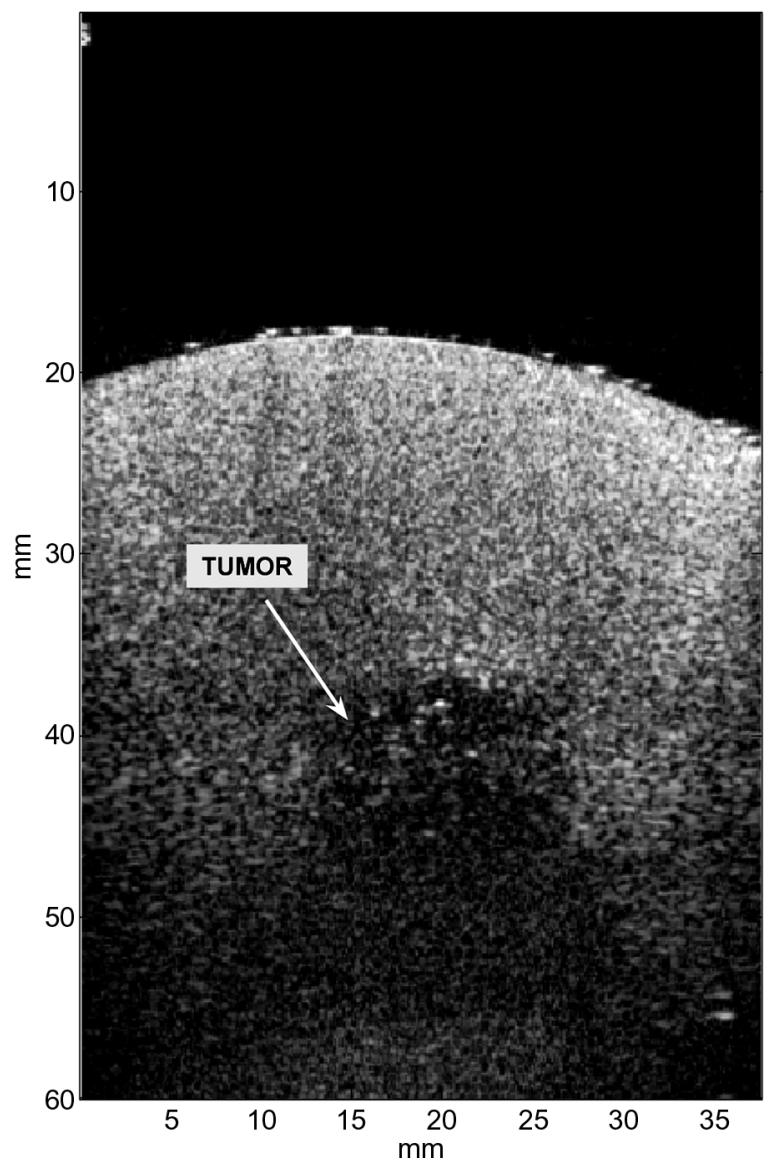

Fig 6. Raw data set showing simulated tumor within tissue-mimicking phantom.

where $S_{i}$ is the mean pixel intensity inside the cyst, $S_{o}$ is the mean pixel intensity outside of the cyst (samples of the intensity coming from same-sized areas), and $\sigma_{o}, \sigma_{i}$ are the standard deviations of the data used to calculate $S_{o}$ and $S_{i}$, respectively. A rectangular area inside the hypoechoic region of the cyst, but not touching the cyst boundary, was chosen for the inside statistics. An area identical in size, next to the cyst but not touching the cyst boundary, was chosen for the outside statistics.

The second image metric is the width of the echo pattern created by the glass beads. This was measured by a distance measuring tool programmed in MATLAB, using on-screen calipers. Ten measurements on the same echo pattern (at different positions, i.e., different diameters) were averaged to produce the measurement. This technique is similar to that which would be employed in a clinical setting.

\section{Results AND DisCUSSION}

\section{A. Images}

An example, raw data set from the first type of phantom, containing a simulated tumor, can be seen in Fig. 6. The reconstructed result, using standard spatial compounding, is shown in Fig. 7, while the reconstructed result using all quality matrices is shown in Fig. 8. The images are created with the same intensity mapping for comparison. The improvement between raw and compounded data is apparent. The improvement between standard compounding and quality-matrix compounding is most noticeable in the middle of the phantom, corresponding 


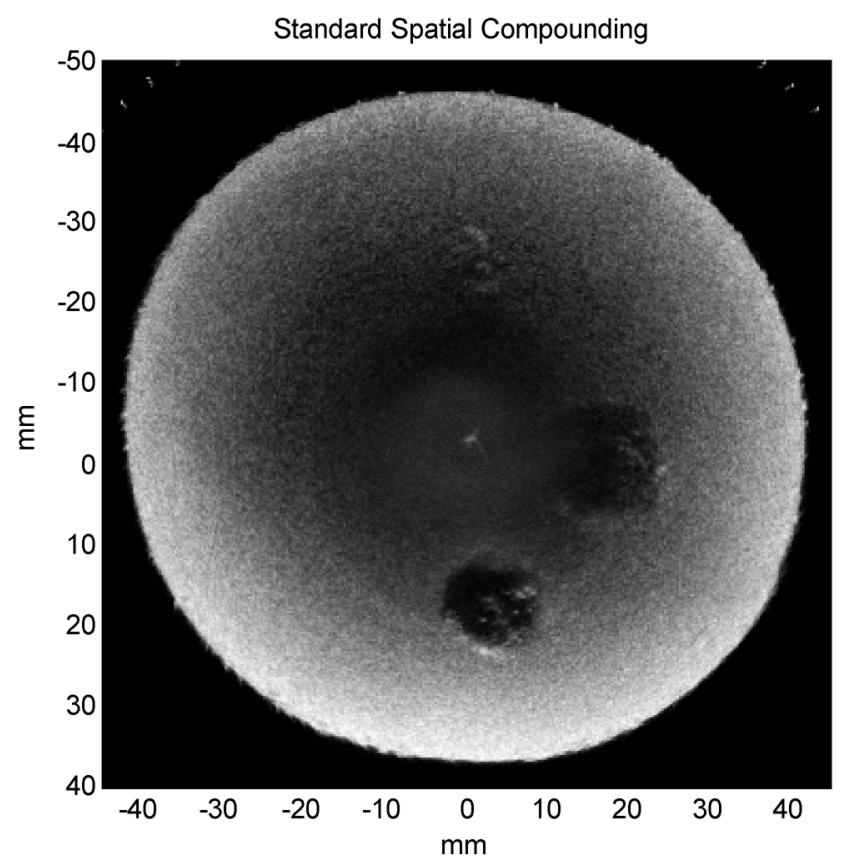

Fig. 7. Reconstructed phantom using standard spatial compounding.

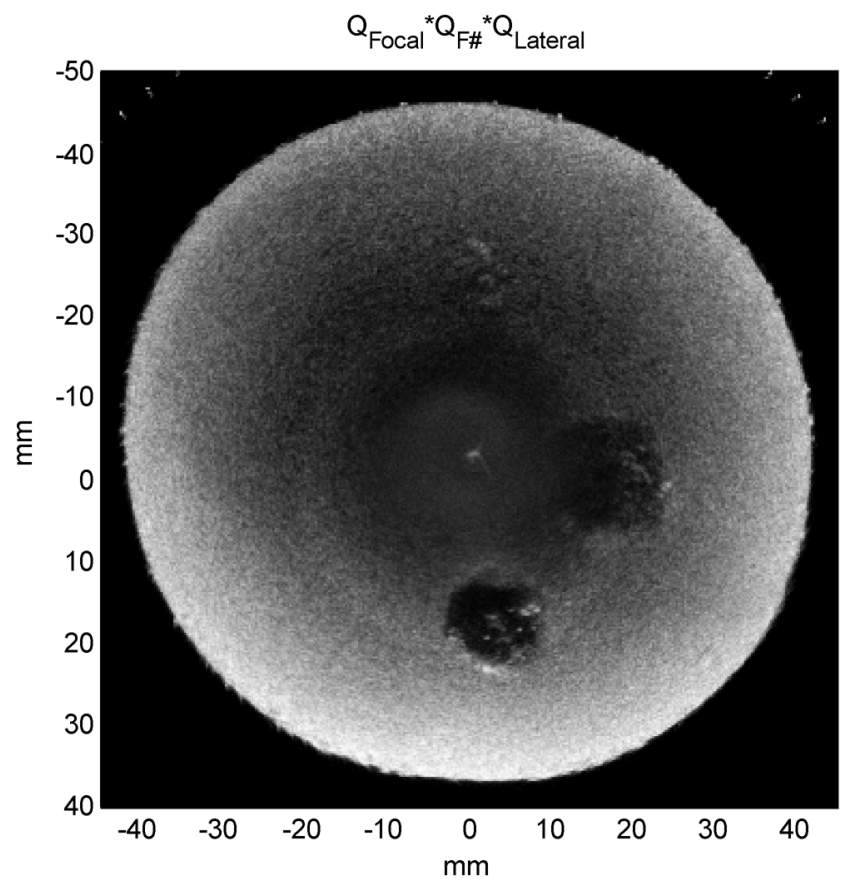

Fig. 8. Reconstructed phantom using all quality matrices.

approximately to where the focal zone is located. An example, raw data set from the second type of phantom, containing glass beads of 1.0- and 2.5-mm size, is shown in Fig. 9. A portion of the reconstruction of the second type of phantom using standard compounding versus all quality matrices, is shown in Fig. 10. The portion chosen was approximately the same region as the raw data set of Fig. 9. The bead targets are easier to distinguish in the reconstruction image than in the raw image.

A comparison of Fig. 7 with Fig. 8 shows similar qualitative results between standard compounding and the new algorithm. In Fig. 10, the difference is more evident in the region of

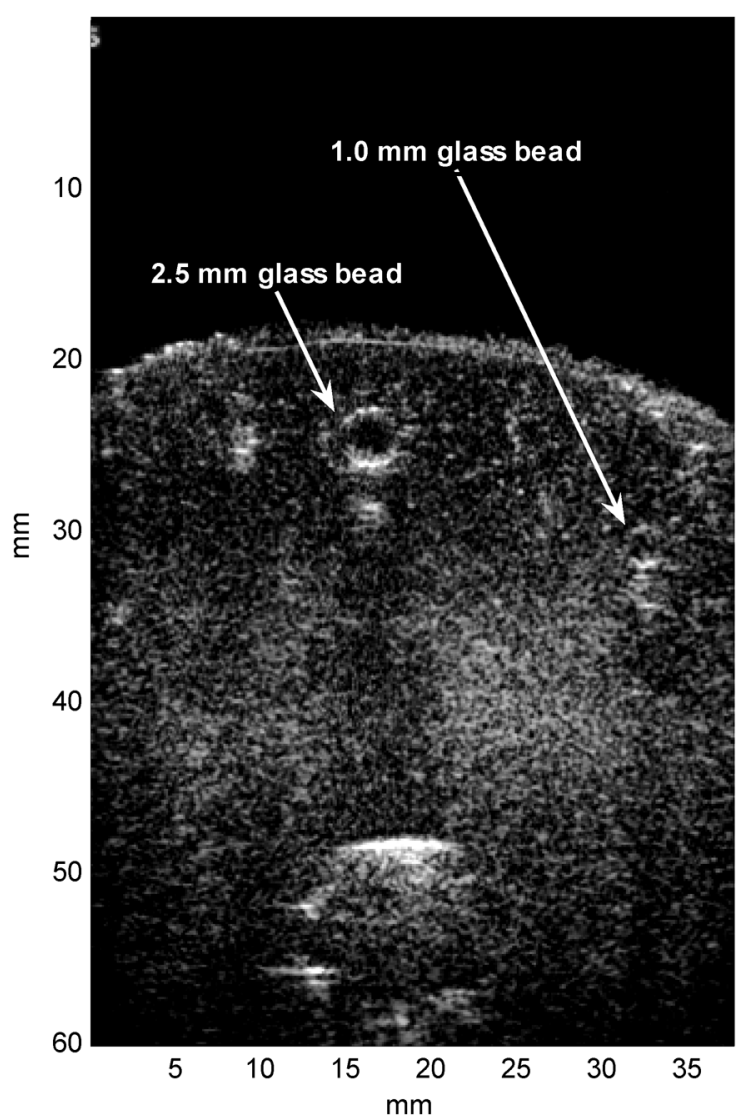

Fig. 9. Raw data set from tissue phantom containing 1.0- and 2.5-mm glass beads.

the image between the glass bead and the bottom of the image. This area is slightly brighter, as it brings out more detail in the speckle pattern. This is explained by the location of the transmit focus, which is at about $-15 \mathrm{~mm}$ on the image scale. Compounding with $\mathrm{Q}_{\text {Focal }}$ as part of the quality matrix results in a greater preference for raw data near the transmit focus. The qualitative appearance of images is an important factor, as most medical images are still evaluated qualitatively by a clinician. In our experiments, we have seen that the qualitative difference between the new algorithm and standard compounding varies. More consistent results are obtained when evaluating the images quantitatively (see Section IV-B).

The effects of azimuthal resolution can be seen in Fig. 11, which is the same phantom as in Fig. 10, but acquired at a vertical distance $1.0 \mathrm{~mm}$ away (deeper) than the slice shown in Fig. 10. The orientation of Figs. 10 and 11 are similar. The $2.5 \mathrm{~mm}$ glass bead at the top of the phantom can faintly be seen in the reconstruction, and another $2.5-\mathrm{mm}$ glass bead is starting to appear in the lower right corner. The $1.0-\mathrm{mm}$ glass bead visible in Fig. 10 is barely seen in Fig. 11.

\section{B. Contrast and Target Size Measurements}

Table III shows the CSR measurements made on a simulated cyst and tumor from the phantom portrayed in Fig. 8. All measurements on raw data were taken at an angle where the feature of interest was centered within the image. The percentage average improvement was calculated by the percentage difference 

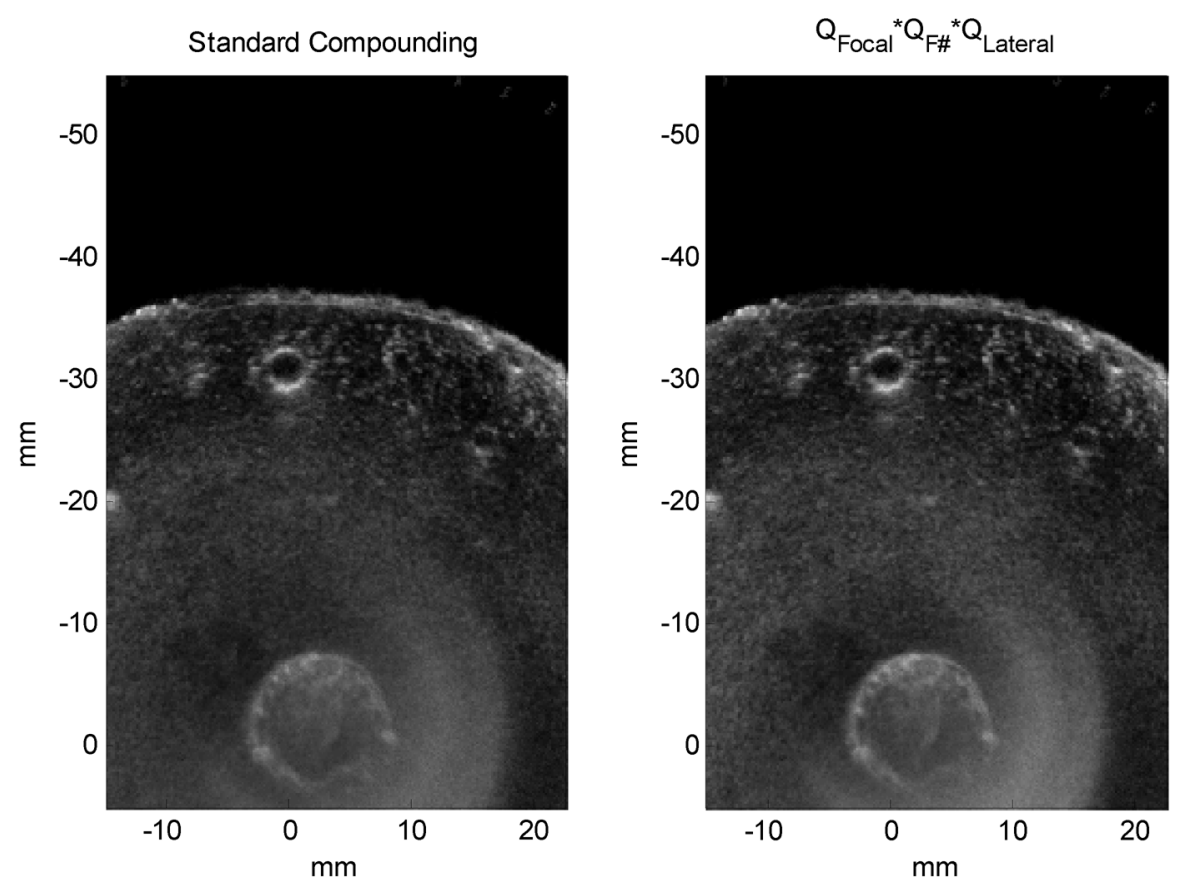

Fig. 10. Reconstructed data set of phantom shown in Fig. 9. (left) Standard compounding. (right) Using all quality matrices.

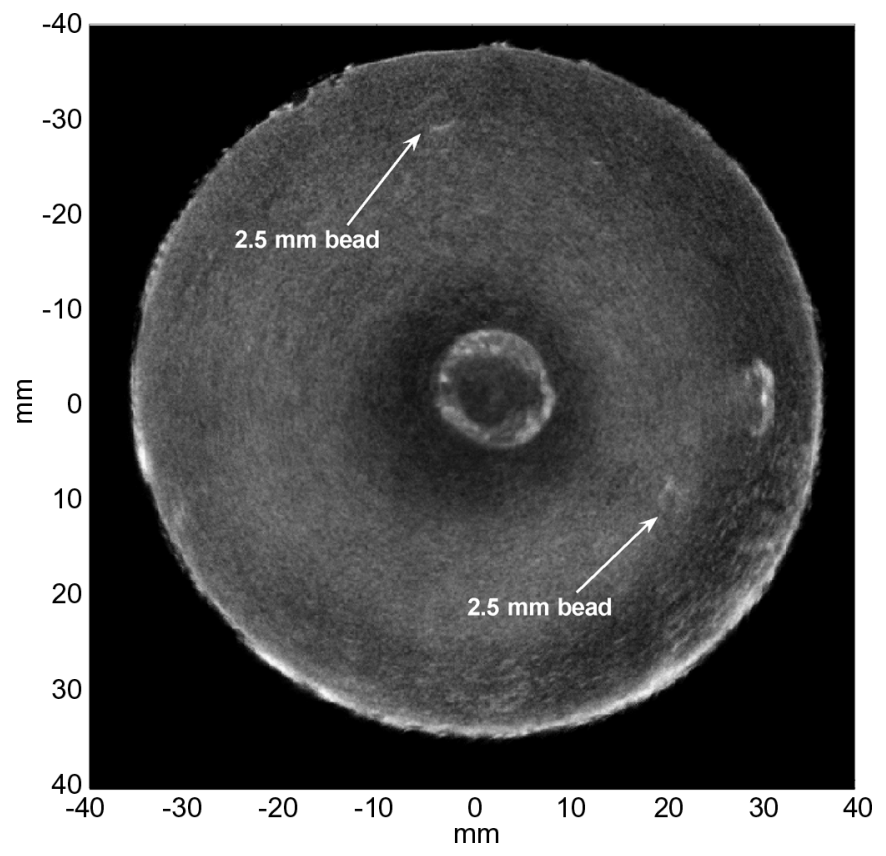

Fig. 11. Reconstructed data of second type of phantom, $1.0 \mathrm{~mm}$ deeper plane than in Fig. 10

of the average CSR values (simulated cyst and tumor) between the quality reconstruction and the raw data.

In Table III, $\mathrm{Q}_{1}, \mathrm{Q}_{\mathrm{focal}}, \mathrm{Q}_{\mathrm{F} \#}$, and $\mathrm{Q}_{\text {Lateral }}$ are the four quality matrices as described in Section II. A large jump in improvement came from standard spatial compounding. Using combinations of the quality matrices in general caused increased improvement in the CSR. However, the improvement is more incremental, and for some cases, standard compounding was better than a particular quality matrix. The best overall improvement ( $60 \%$ increase in CSR) occurred when all quality matrices were used, which indicates that a combination of factors is necessary to achieve the best effect.

It should be noted that reconstruction is employed such that nonoverlapping segments of raw data are not averaged together. That is, the raw data sets overlap to a greater extent near the center of the reconstruction and to a lesser extent near the outside of the image. The weighted averaging takes into account how many pixels actually overlap in each point in the image. This avoids the problem of decreasing brightness near the outside of the final image.

For small-diameter targets, the compounding method shows much-improved results, especially in edge definition. Fig. 12 shows a side-by-side close-up view of a $2.5-\mathrm{mm}$ bead, comparing the raw data, standard compounding, and reconstruction with all quality matrices. Again, the largest jump in improvement is due to compounding in general, with a slight improvement in edge definition by using quality matrices. Table IV shows the results of taking ten measurements each of the raw, standard compounding, and all quality matrix compounding images of a close-up view of the bead. The difference in measurements is statistically significant between raw images and compounded images (double-sided p-value $\sim 0.02$ ), but not statistically significant between standard compounding and new compounding methods (double-sided p-value $~ 0.80$ ). However, the ease in taking measurements is greatly aided by having clearer boundaries. The result of an overall measured bead size $(\sim 3.4 \mathrm{~mm})$ being greater than the $2.5-\mathrm{mm}$ actual size is most likely due to blurring effects of the bead boundary due to the PSF of the system.

Circular artifacts can also be seen in the compounded image. When designing the quality matrices, no attempt was made to equalize gain throughout the reconstructed image. Therefore, the final image may show depth-dependent gain, which manifests as alternating circular bright and dark variations from 
TABLE III

CSR MEASurements on Gelatin/OIL Phantom

\begin{tabular}{lccc}
\hline \hline Quality Matrices Used & $\begin{array}{c}\text { Cyst } \\
\text { CSR }\end{array}$ & $\begin{array}{c}\text { Tumor } \\
\text { CSR }\end{array}$ & $\begin{array}{c}\text { Average Improvement } \\
\text { (\%) }\end{array}$ \\
\hline None (raw data) & 1.82 & 1.93 & - \\
Q1 (Standard spatial & 2.24 & 3.32 & 48.4 \\
$\quad$ compounding) & 2.36 & 3.24 & 49.5 \\
QFocal & 2.53 & 3.22 & 53.2 \\
QF\# $_{\text {QLateral }}$ & 2.32 & 3.37 & 51.7 \\
QFocal $^{*}$ QF\# $_{\text {QFoal }}$ Q $_{\text {Lateral }}$ & 2.56 & 3.16 & 52.4 \\
QF\# $_{\text {Lateral }}^{\text {QFocal }}$ & 2.39 & 3.33 & 52.4 \\
\hline \hline
\end{tabular}

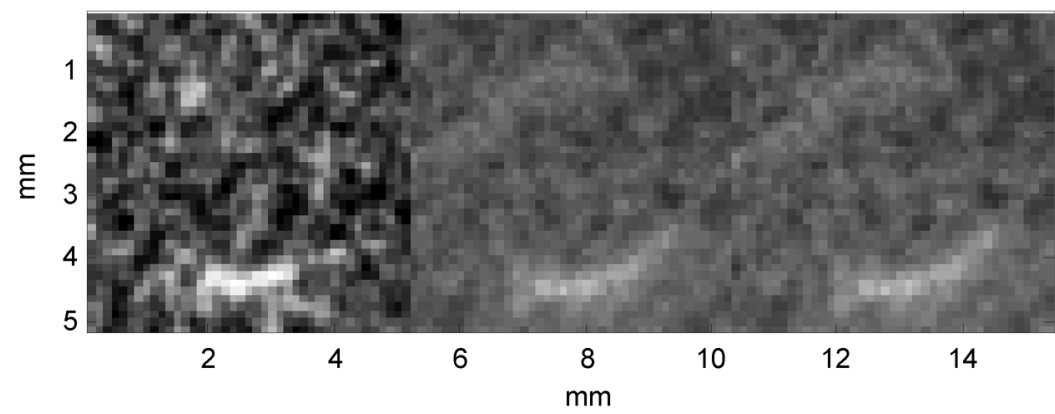

Fig. 12. Side-by-side close-up view of 2.5-mm glass bead, raw and reconstructed data. (left) Raw data. (center) Standard compounding reconstruction. (right) Reconstruction with all quality matrices.

TABLE IV

DiAMETER MEASUREMENTS ON 2.5-mm GLASS BEAD

\begin{tabular}{lcc}
\hline \hline Quality Matrices Used & Mean $(\mathrm{mm})$ & Std $(\mathrm{mm})$ \\
\hline None (raw data) & 3.55 & 0.19 \\
Q1 $_{1}$ (Standard spatial compounding) & 3.38 & 0.19 \\
QFocal ${ }^{*}$ QF* $^{*}$ QLateral & 3.36 & 0.15 \\
\hline \hline
\end{tabular}

the mean. As noted in Section II, this artifact is not related to the gain compensation that had already been applied by the ultrasound machine in forming the raw image data. The reconstruction algorithms described here assume that the raw data sets have already been compensated for signal loss due to attenuation.

\section{Conclusions and Future Work}

These results suggest that designed quality matrices have the potential to improve image quality parameters of contrast and target edge definition over conventional beamforming at minimal computational expense. Standard spatial compounding gives a large improvement in image quality, which can be modestly improved by designed matrix weighting. The objectives of introducing the method and comparing its performance to standard spatial compounding have been achieved.

There are several improvements which can potentially increase the image quality, and should be considered for further work. A significant drawback is the need to work with JPEG images in which much of the dynamic range of the internal ultrasound data has been lost. However, access to the internal data requires an interface to the commercial machine, which is not yet available to our group. Next, the circular artifacts may be corrected by experimenting with quality matrices created with different (e.g., nonlinear) functions. As previously noted, the creation (especially the shape) of quality matrices is somewhat subjective, and more mathematical rigor could be applied to predicting what types of functional forms will have optimal effects. In particular, it is hypothesized that quality matrices taking into account other position-dependent aspects of the point spread function (PSF), such as tail shape and axial length, would be superior to those studied here. Also, although resolution was partially addressed by target boundary definition, a rigorous comparison of the minimum distance resolvable between two small (smaller than the glass beads used here) targets is needed. Another factor to be considered in future work is the number of images used for reconstruction, i.e., how the angular spacing of the raw data sets affects resolution and contrast. Finally, this paper did not attempt to correct for the effects of phase aberration, which are known to affect breast imaging [16]. Addressing these issues is the focus of future research.

\section{ACKNOWLEDGMENT}

The authors wish to thank S. Minchow for his help in designing and machining the custom experimental setup, and G. Ogden for her work in providing photographs of the experimental setup and tissue-mimicking phantoms.

\section{REFERENCES}

[1] U.S. Preventive Services Task Force, Statement on Mammography Screening. 2002 [Online]. Available: http://www.ahrq.gov/clinic/ 3rduspstf/breastcancer/brcanrr.htm

[2] National Cancer Institute, Statement on Mammography Screening 2002 [Online]. Available: http://www.nci.nih.gov/newscenter/mammstatement $31 \mathrm{jan} 02$

[3] J. Y. Kwak, E.-K. Kim, J. K. You, and K. K. Oh, "Variable breast conditions: Comparison of conventional and real-time compound ultrasonography," J. Ultrasound Med., vol. 23, pp. 85-96, 2004. 
[4] P. Lasaygues, D. Tanne, S. Mensah, and J. P. Lefebvre, "Circular antenna for breast ultrasonic diffraction tomography," Ultrason. Imag., vol. 24, no. 3, pp. 177-189, 2002.

[5] C. B. Burckhardt, "Speckle in ultrasound B-mode scans," IEEE Trans. Sonics Ultrason., vol. SU-25, no. 1, pp. 1-6, 1978.

[6] R. R. Entrekin, B. A. Porter, H. H. Sillesen, A. D. Wong, P. L. Cooperberg, and C. H. Fix, "Real-time spatial compound imaging: Application to breast, vascular, and musculoskeletal ultrasound," Semin. Ultrasound CT MR, vol. 22, no. 1, pp. 50-64, 2001.

[7] S. Huber, M. Wagner, M. Medl, and H. Czembirek, "Real-time spatial compound imaging in breast ultrasound," Ultrasound Med. Biol., vol. 28, no. 2, pp. 155-163, 2002.

[8] J. C. Lacefield, W. C. Pilkington, and R. C. Waag, "Comparisons of lesion detectability in ultrasound images acquired using time-shift compensation and spatial compounding," IEEE Trans. Ultrason. Ferroelectr. Freq. Control, vol. 51, no. 12, pp. 1649-1659, Dec. 2004.

[9] L. Nock, G. E. Trahey, and S. W. Smith, "Phase aberration correction in medical ultrasound using speckle brightness as a quality factor," $J$. Acoustical Soc. Amer., vol. 85, no. 5, pp. 1819-1833, 1989.

[10] E. L. Madsen, J. A. Zagzebski, and G. R. Frank, "Oil-in-gelatin dispersions for use as ultrasonically tissue-mimicking materials," Ultrasound in Med. Bio., vol. 8, no. 3, pp. 277-287, 1982.
[11] E. L. Madsen, G. A. Frank, T. A. Krouskop, T. Varghese, F. Kallel, and J. Ophir, "Tissue-mimicking oil-in-gelatin dispersions for use in heterogeneous elastography phantoms," Ultrason. Imag., vol. 25, pp. $17-38,2003$.

[12] E. L. Madsen, E. Kelly-Fry, and G. A. Frank, "Anthropomorphic phantoms for assessing systems used in ultrasound imaging of the compressed breast," Ultrasound in Med. Bio., vol. 14, pp. 183-201, 1988, Sup. 1.

[13] E. L. Madsen, J. A. Zagzebski, R. A. Banjavie, and R. E. Jutila, "Tissue mimicking materials for ultrasound phantoms," Med. Phys., vol. 5, no. 5, pp. 391-394, 1978.

[14] M. S. Patterson and F. S. Foster, "The improvement and quantitative assessment of B-mode images produced by an annular array/cone hybrid," Ultrason. Imag., vol. 5, no. 3, pp. 195-213, 1983.

[15] S. W. Smith and R. F. Wagner, "Ultrasound speckle size and lesion signal to noise ratio: Verification of theory," Ultrason. Imag., vol. 6, pp. $174-180,1984$.

[16] P. D. Freiburger, D. C. Sullivan, B. H. LeBlanc, S. W. Smith, and G. E Trahey, "Two dimensional ultrasonic beam distortion in the breast: In vivo measurements and effects," Ultrason. Imag., vol. 14, pp. 398-414, 1992. 